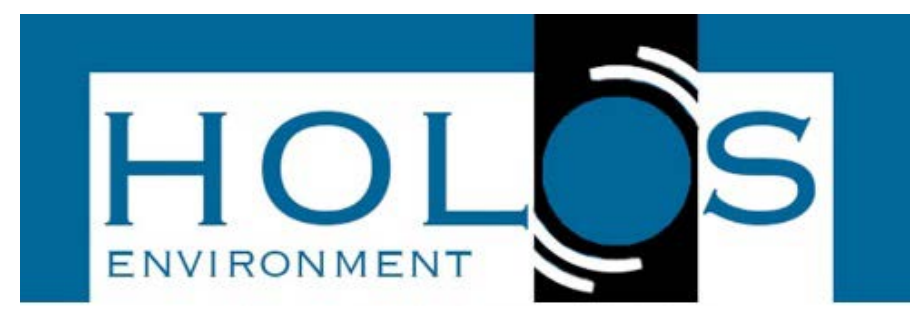

\title{
MODELAGEM ESPACIAL DA EVAPOTRANSPIRAÇÃO E PRODUTIVIDADE HÍDRICA NA PORÇÃO PAULISTA DO AFLORAMENTO DO AQUÍFERO GUARANI ENTRE 2013 E 2015
}

\author{
SPATIAL MODELING OF EVAPOTRANSPIRATION AND WATER PRODUCTIVITY IN THE \\ OUTCROP AREA OF THE GUARANI AQUIFER IN SÃO PAULO STATE BETWEEN 2013 AND \\ 2015
}

\author{
Cesar de Oliveira Ferreira Silva1; Rodrigo Lilla Manzione2; Antônio Heriberto de Castro \\ Teixeira $^{3}$
}

Artigo recebido em: 13/03/2018 e aceito para publicação em: 17/05/2018.

DOI: http://dx.doi.org/10.14295/holos.v18i2.12266

Resumo: Este artigo teve como objetivo avaliar a distribuição espacial e temporal dos parâmetros da produtividade hídrica (evapotranspiração e produção de biomassa) para a porção paulista do Aquífero Guarani entre os anos de 2013 e 2015, buscando verificar o impacto da estiagem de 2014 na região. O algoritmo utilizado para calcular a evapotranspiração foi o SAFER (Simple Algorithm For Evapotranspiration Retrieving) e a equação de Monteith para estimar os parâmetros da produção de biomassa. Foram utilizados dados de estações agrometeorológicas e imagens digitais sem nuvens do satélite MODIS de dias julianos iguais entre 2013 e 2015. Em valores médios, para toda área paulista do afloramento do Aquífero Guarani, ocorreu diminuição da evapotranspiração e produção de biomassa nos anos de estiagem (2014-5). A produtividade hídrica, quando tomada em referência uma série histórica que permita a análise de tendência, é um potencial indicador de estresse hídrico de vegetação e culturas. A queda expressiva na evapotranspiração em 2014 e 2015 é uma evidência de impactos na recarga de aquífero e diminuição no nível freático. Foram encontradas evidências de que a estiagem teve maior impacto na região rural ao norte do afloramento paulista do Aquífero Guarani (região de Ribeirão Preto) em comparação à região do Paranapanema, sendo uma possível explicação para isso o fato da primeira ter predomínio de plantio de cana-de-açúcar e a segunda de Eucaliptos. Essas informações espaciais geram subsídios para tomada de decisão em gestão ambiental regional.

Palavras-chave: Afloramento do Aquífero Guarani. Evapotranspiração real. Produção primária de biomassa. Sensoriamento remote. MODIS.

Abstract: This research aims to evaluate the spatial and temporal distribution of of water productivity (evapotranspiration and biomass production) for São Paulo Guarani Aquifer Outcrop between 2013 and 2015, in order to evaluate the impact of the drought of 2014 in the region. The algorithm used to calculate evapotranspiration was SAFER (Simple Algorithm For Evapotranspiration Retrieving) and the Monteith equation to estimate the parameters of biomass production. Were used data from agrometeorological stations and digital images without clouds from MODIS satellite of the same julian days between 2013 and 2015. In

\footnotetext{
${ }^{1}$ Universidade Estadual Paulista "Júlio de Mesquita Filho", Faculdade de Ciências Agronômicas, Botucatu, SP, Brasil. Email: (cesaroliveira.f.silva@gmail.com). ORCID: http://orcid.org/0000-0002-5152-6497

2 Universidade Estadual Paulista "Júlio de Mesquita Filho", Faculdade de Ciências e Engenharia, Tupã, SP, Brasil. Email: (manzione@tupa.unesp.br). ORCID: http://orcid.org/0000-0002-0754-2641

${ }^{3}$ Embrapa Tabuleiros Costeiros, Aracaju, Sergipe, SE. Email: (heriberto.teixeira@embrapa.br)
} 
average values, for all the São Paulo area of the outcrop of the Guarani aquifer, there was a decrease in evapotranspiration and biomass production in the drought years (2014-2015). Water productivity, when taken in reference to a historical series that allows a trend analysis, is an indicator of water stress of vegetation and crops. The significant decrease in evapotranspiration in 2014 and 2015 is an evidence of impacts on aquifer recharge and decrease in the water table. Evidence was found that drought had a greater impact in the rural region north of the outcrop of the Guarani Aquifer (Ribeirão Preto region) compared to the Paranapanema region, being a possible explanation for this the fact that the first one has predominance of sugarcane and the second one of Eucalyptus. This spatial information generates subsidies for decision making in regional environmental management.

Keywords: Guarani Aquifer outcrop. Actual evapotranspiration. Biomass primary production. Remote sensing. MODIS.

\section{INTRODUÇÃO}

Os recursos hídricos subterrâneos são fundamentais na conservação da dinâmica do sistema hidrológico, exigindo ações de planejamento para assegurar que seu uso, que muitas vezes é feito de maneira contínua, respeite as oscilações sazonais de recarga (SILVA e MANZIONE, 2016), as características ambientais (REISSLER e MANZIONE, 2016) e o comportamento natural dos aquíferos (SANTAROSA e MANZIONE, 2017), impedindo impactos negativos à quantidade e qualidade da água. A necessidade de informações ambientais para setores envolvidos com o agronegócio e com o gerenciamento ambiental, e os altos custos de investimento e manutenção em equipamentos para sua obtenção, impulsionaram a popularização de técnicas de sensoriamento remoto orbital para conduzir mapeamentos (TEIXEIRA et al., 2017, 2016), tornando possível um diagnóstico espacial de forma mais barata, rápida e proporcionando ferramentas para o planejamento integrado entre recursos ambientais, agrícolas e sociais em diversas escalas (TEIXEIRA et al., 2013).

O Sistema Aquífero Guarani (SAG) constitui uma importante reserva mundial de água subterrânea. No Brasil estima-se que 1.443 municípios se localizam na área do SAG (IBGE, 2018; MANZIONE et al, 2015), constituindo uma área de grande importância econômica e apresentando diversos usos como urbano, industrial e rural. Assim, existe a necessidade de realizar predições seguras a respeito do comportamento do SAG, auxiliando a tomada de decisão no planejamento dos recursos hídricos (MANZIONE et al, 2016, 2015). Um melhor conhecimento dos processos que ocorrem no sistema solo-plantaágua-atmosfera visa o planejamento e o uso eficiente da água, além da redução dos impactos antrópicos negativos no uso da água. 
A evapotranspiração (ET) é uma das mais importantes variáveis no ciclo hidrológico, fazendo a ligação entre energia, disponibilidade hídrica e clima. A mudança de cobertura e uso do solo gera impactos na interação biosfera-atmosfera, afetando variáveis climáticas importantes. Envolve todos os processos de evaporação e transpiração que ocorrem simultaneamente em uma superfície vegetada, onde manifestam-se diferentes fontes de vapor d'água, como a água livre na superfície, o solo superficial úmido, a água da chuva interceptada pelos dosséis e a transpiração das plantas (NAVA e MANZIONE, 2015).

Tendo em vista que tais parâmetros são passíveis de monitoramento por sensoriamento remoto, é possível avaliar como situações passadas alteraram-nas. A maior parte da estação chuvosa de 2014 transcorreu com valores de chuva inferiores à média histórica sobre a porção sudeste do país, incluindo o Estado de São Paulo (NOBRE et al., 2015; DOBROVOLSKI e RATTIS, 2015; ESPINOZA et al., 2014). A combinação dos baixos índices pluviométricos, o grande crescimento da demanda de água e o ineficiente gerenciamento desse recurso geraram uma crise hídrica durante os anos 2014 e 2015.

Este estudo teve como objetivo avaliar a distribuição espacial e temporal dos parâmetros de produtividade hídrica (evapotranspiração e produção de biomassa) para a porção paulista do Aquífero Guarani durante os anos de 2013, 2014 e 2015, buscando verificar o impacto da estiagem de 2014 e sua capacidade de subsidiar ações de planejamento e gestão ambiental em escalas estaduais e regionais.

\section{MATERIAIS E MÉTODOS}

A área de estudo é composta por 106 municípios do estado de São Paulo situados sobre o afloramento do Aquífero Guarani. A Figura 1 expõe a distribuição espacial do uso do solo no afloramento do Aquífero Guarani em 2016, segundo Mapbiomas (2018), e sua localização no Estado de São Paulo. O município de Ribeirão Preto é o único município com mais de 500 mil habitantes, tendo 547.417 habitantes. Os municípios de Piracicaba (358.108 habitantes), Franca (332.109 habitantes), São Carlos (220.425 habitantes), Araraquara (198.079 habitantes), Rio Claro (191.135 habitantes), Jaú (125.469 habitantes), Botucatu (120.800 habitantes) e Araras (108.689 habitantes) representam importantes municípios da região (IBGE, 2018). Existe uma crescente utilização das águas subterrâneas para abastecimento público nessa área, sendo que aproximadamente $57 \%$ dos municípios 
da região do afloramento do Aquífero Guarani utilizam o manancial subterrâneo como fonte de abastecimento (ALBUQUERQUE FILHO et al, 2011).

Para estimativa da evapotranspiração foram utilizadas as bandas espectrais do visível e infravermelho próximo de imagens do satélite MODIS (Moderate Resolution Imaging Spectroradiometer) com resolução espacial de $250 \mathrm{~m}$ de dias julianos iguais entre 2013 e 2015 e sem nuvens. Dados de radiação solar global $\left(R_{G}\right)$, temperatura do $\operatorname{ar}\left(T_{a}\right)$ e evapotranspiração de referência $\left(E T_{0}\right)$ foram obtidos de estações agrometeorológicas do INMET (Instituto Nacional de Meteorologia).

Figura 1 - Porção paulista do Afloramento do Aquífero Guarani e seu uso do solo em 2016 (MAPBIOMAS, 2018)

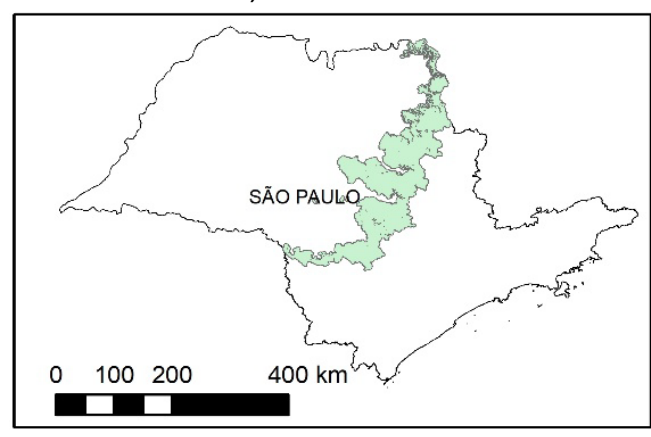

\section{Uso do Solo}

Agricultura Anual
Agricultura Perene
Corpos Hidricos
Área Urbana
Mineração
Mosaico de Ocupações
Não Observado
Vegetação Natural
Pastagem
Silvicultura
Solo Exposto
Limites Políticos Municipais

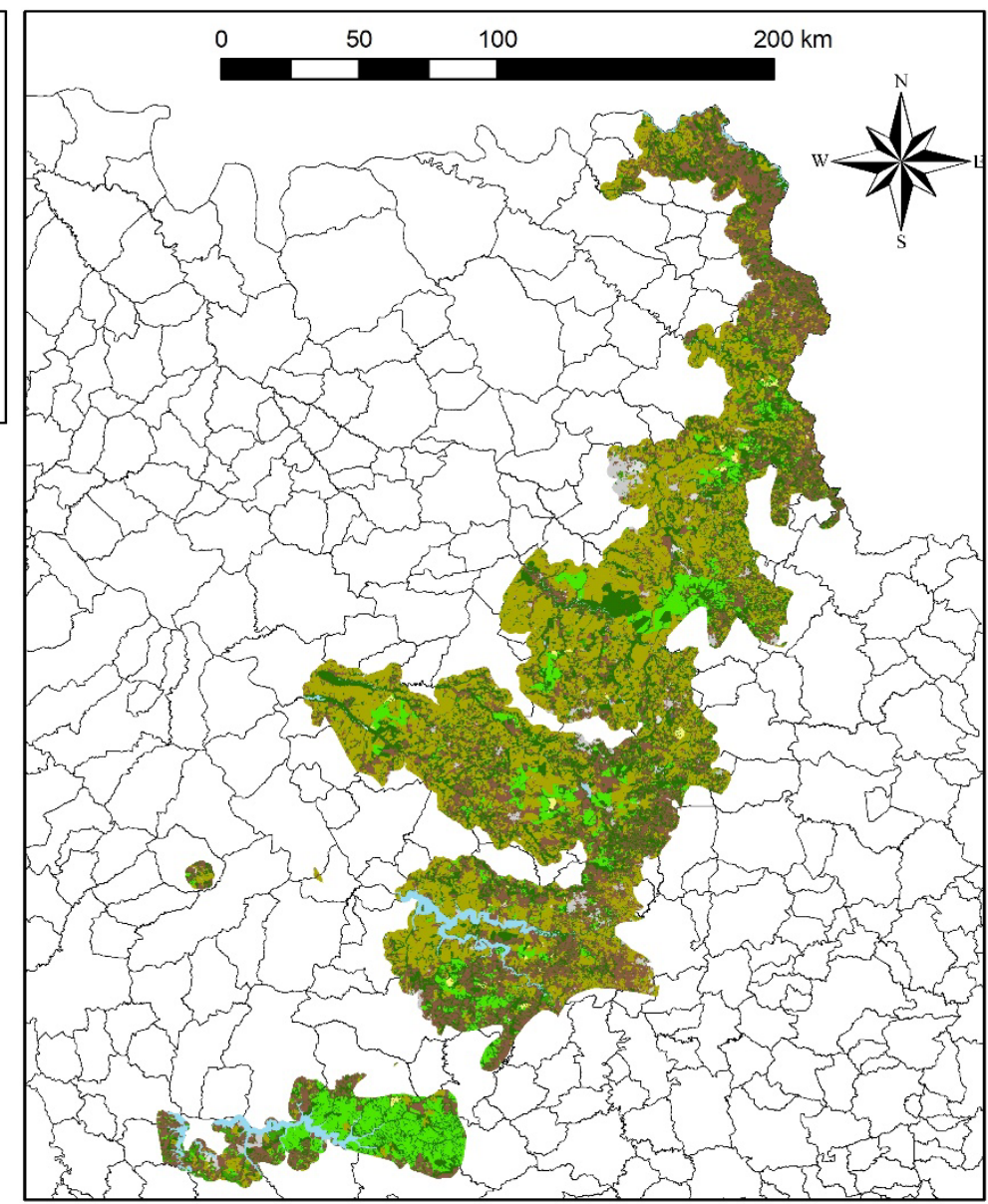

Seguindo a metodologia de Teixeira (2017, 2016, 2013) os parâmetros biofísicos obtidos via sensoriamento remoto foram o albedo de superfície $\left(\alpha_{0}\right)$, componentes de energia, como o saldo de radiação $\left(R_{n}\right)$, fluxo de calor latente $(\lambda E)$, o fluxo de calor sensível 
(H) e o fluxo de calor no solo (G); Índice de Vegetação da Diferença Normalizada - NDVI e a radiação de onda longa emitida pela atmosfera ( $\left.R L_{a t m}\right)$.

O $R_{n}$ na escala diária foi obtido pelo saldo diário $(24 \mathrm{~h})$ de radiação de onda curta, por meio da equação de Slob (Eq. 1) (TEIXEIRA et al., 2010).

$$
R_{n}=\left(1-\alpha_{0}\right) R_{G}-a_{L} \tau_{s w}
$$

Onde $\alpha_{0}$ é o albedo de superfície, $a_{L}$ é o coeficiente de regressão do saldo de radiação de ondas longas e $\tau_{s w}$ é a transmissividade atmosférica (BRUIN, STRICKER, 2000).

Uma particularidade dessa metodologia foi a obtenção da temperatura de superfície pelo método residual (TEIXEIRA, LEIVAS e SILVA, 2016), derivado da Equação de Planck. Nesse método a temperatura de superfície foi obtida pela Equação 2:

$$
T_{0}=\sqrt[4]{\frac{R_{G}-\alpha_{0} R_{G}+\varepsilon_{A} \sigma T_{a}^{4}-R_{n}}{\varepsilon_{S} \sigma}}
$$

Sendo $\varepsilon_{A}$ e $\varepsilon_{S}$ respectivamente as emissividades atmosférica e superficial e $\sigma$ a constante de Stefan-Boltzmann $\left(5.67 \cdot 10^{-8} \mathrm{~W} \mathrm{~m}^{-2} \mathrm{~K}^{-4}\right)$.

Quando o NDVI < 0, indicativo da presença de corpos hídricos, foi utilizado o conceito de evapotranspiração de equilíbrio (RAUPACH, 2001) transformando unidades de energia em $\mathrm{mm} \mathrm{dia}^{-1}$ (TEIXEIRA et al., 2017, 2016):

$$
\lambda E=\frac{\Delta\left(R_{n}-G\right)}{\Delta+\gamma}
$$

Onde $\Delta\left(\mathrm{kPa}^{\circ} \mathrm{C}^{-1}\right)$ é a curva de saturação de vapor de água, $G\left(\mathrm{MJ} \mathrm{m}^{-2}\right.$ day $^{-1} \mathrm{o}$ fluxo de calor no solo e $\gamma\left(\mathrm{kPa}^{\circ} \mathrm{C}^{-1}\right)$ a constante psicométrica. A densidade de fluxo de calor no solo $(G)$ foi estimada a partir da sua relação com o saldo de radiação à superfície da cultura (COAGUILA et al, 2017; TEIXEIRA et. al., 2017) expressa na Equação 4: 


$$
\frac{G}{R_{n}}=3.98 \cdot \exp \left(-31.89 \cdot \alpha_{0}\right)
$$

Calculou-se a relação $\frac{E T}{E T_{0}}$ diários seguindo a Equação 5.

$$
\frac{E T}{E T_{0}}=\exp \left[a+b\left(\frac{T_{0}}{\alpha_{0} . N V D I}\right)\right]
$$

Onde a e b são coeficientes de regressão calibrados para a região de estudo. Teixeira et al, 2009 utiliza, respectivamente, os valores de 1,9 e -0.008 para a região do semiárido nordestino. Coaguila et al (2017) utiliza para a região do noroeste paulista os valores de 1 e 0.008, sendo esses os parâmetros utilizados no presente trabalho.

O produto da razão obtida na Equação 6 pelo valor diário da evapotranspiração de referência é a evapotranspiração real $\left(E T_{A}\right)$ diária (Eq. 6):

$$
E T_{A}=\frac{E T}{E T_{0}} E T_{0}
$$

Onde $E T_{0}$ é a evapotranspiração de referência, obtida por interpolação de dados das estações agrometeorológicas.

A produção de biomassa $\left(\mathrm{kg} \mathrm{ha}^{-1} \mathrm{dia}^{-1}\right)$ foi calculada usando o modelo de Monteith (TEIXEIRA et al, 2015) (Eq. 7).

$$
B I O=\varepsilon_{\max } \frac{E T}{E T_{0}} A P A R \cdot 0.864
$$

Onde $\varepsilon_{\max }$ é a eficiência máxima no uso da radiação por parte da vegetação (COAGUILA et al, 2017) e APAR a radiação fotosseticamente ativa absorvida, que foi obtida através da relação com o NDVI e a radiação solar global (TEIXEIRA et al, 2015) (Eq. 8).

$$
A P A R=(1.26 \cdot N D V I-0.26)\left(0.44 \cdot R_{G}\right)
$$


A produtividade hídrica $\left(\mathrm{kg} \mathrm{m}^{-3}\right)$, que quantifica a biomassa produzida por cada metro cúbico de água consumida pela evapotranspiração, indicando a eficiência do uso da água pela planta (TEIXEIRA et al, 2009; TEIXEIRA, LEIVAS e SILVA, 2016; COAGUILA et al, 2017) foi calculada através da equação 9 (TEIXEIRA et al, 2013):

$$
P A=\frac{B I O}{E T}
$$

O procedimento metodológico utilizado neste trabalho é apresentado de forma esquemática no fluxograma da Figura 2.

Figura 2 - Fluxograma metodológico de modelagem da produtividade hídrica a partir da evapotranspiração real e produção de biomassa

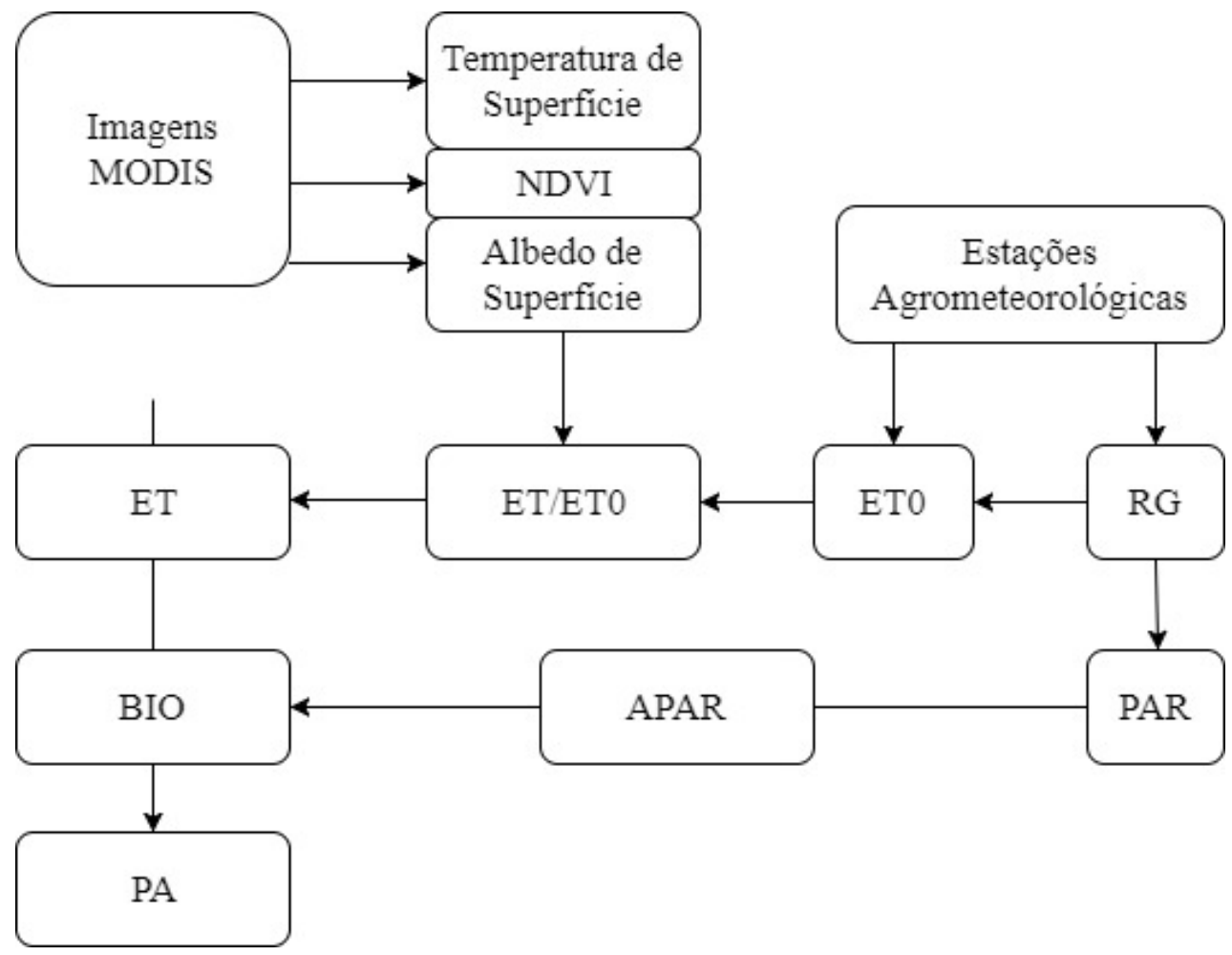

\section{RESULTADOS E DISCUSSÕES}

Os resultados obtidos são apresentados na Tabela 1 e na Figura 3. Na Tabela 1 dividem-se, geograficamente, os resultados da evapotranspiração atual entre área urbana (Urb), área rural na região norte do afloramento do Aquífero Guarani, reconhecida como a 
macrorregião de Ribeirão Preto (RurRP) e área rural na região sul do afloramento do Aquífero Guarani, reconhecida como a macrorregião de Paranapanema (RurPA). Os resultados da produção primária de biomassa e produtividade hídrica são apresentados apenas na região rural do afloramento paulista do Aquífero Guarani, já que são parâmetros intrinsecamente do meio agrícola. A Figura 3 apresenta as médias globais da área de estudo.

A área urbana apresenta a menor evapotranspiração atual. As áreas rurais do Paranapanema apresentam maior taxa de evapotranspiração (entre 20 e 30\%) quando comparada à região de Ribeirão Preto. As extensas áreas de irrigação na região sul do afloramento paulista do Aquífero Guarani (ROMAGNOLI, MANZIONE, 2016) e o predomínio da silvicultura e plantio de Eucaliptos (MAPBIOMAS, 2018; ALBUQUERQUE FILHO et al, 2011), em detrimento a da produção canavieira na região de Ribeirão Preto (MAPBIOMAS, 2018; INPE, 2013; ALBUQUERQUE FILHO et al, 2011), resultam em maiores taxas de evapotranspiração. Teixeira, Leivas e Ronquim (2016) obtiveram uma faixa similar de amplitude (0,6 a $4 \mathrm{~mm})$ de evapotranspiração nas áreas de plantio de cana de açúcar no Norte e Noroeste paulista entre 2011 e 2012, também utilizando imagens digitais do satélite MODIS, enquanto Rodrigues et al (2011) destacam que plantações de Eucaliptos tem alto coeficiente de cultivo empírico, variando entre $154 \%$ e $257 \%$ da evapotranspiração de referência, enquanto a cana atinge no máximo $120 \%$, mostrando coerência com o diagnóstico obtido pelo modelo SAFER na região do afloramento do Aquífero Guarani.

A tendência dos valores de produção primária de biomassa é semelhante a a evapotranspiração, apresentando diferenças médias entre 15\% e 25\%. A produtividade hídrica mostrou maior diferença percentual entre as duas regiões, variando de $25 \%$ a 40\%, influenciado pelas grandes áreas irrigadas por pivôs centrais (ANA, 2016), que são melhor detectáveis à resolução espacial do satélite MODIS do que áreas irrigadas convencionalmente (TEIXEIRA et al, 2015). O ano de 2015 apresenta maior amplitude de valores de evapotranspiração e produção de biomassa, pelo fato de apresentar os menores valores mínimos dos três anos. A tendência temporal mantem-se nos três anos, havendo um período de estiagem prolongada entre os dias julianos 200 e 300.

Analisando toda a área estudada (Figura 3), em valores médios, o impacto da estiagem anormal de 2014-2015 foi verificado por apresentarem menores valores em seus 
índices, mesmo que seguindo a tendência sazonal do ano de 2013. Nota-se que a produção de biomassa acompanha a queda na evapotranspiração, formando curvas similares, porém em menor ordem de magnitude. Esses resultados evidenciam que a metodologia apresentada é capaz de detectar, regionalmente, tendências de parâmetros agroambientais de forma espacializada. O conhecimento de parâmetros da vegetação como evapotranspiração, produção primária de biomassa e produtividade hídrica que possibilitam que ações de gestão ambiental e intervenções emergenciais ou preventivas sejam feitas, melhorando a tomada de decisão em sistemas hidroagrícolas, levando-se em conta que o satélite MODIS tem resolução temporal diária, ou seja em resolução temporal suficiente para esse tipo de aplicação.

A Figura 4 compara a evapotranspiração atual, produção primária de biomassa e produtividade hídrica no $273^{\circ}$ dia juliano nos três anos de estudo, exemplificando espacialmente a variabilidade detectada nesses parâmetros, sendo o dia juliano 273 de 2015 com menor evapotranspiração atual e produção de biomassa. Essa tendência foi observada em toda a série temporal desse estudo, em que a queda na evapotranspiração atual foi acompanhada pela produção de biomassa menor. A produtividade hídrica, no entanto, teve seus maiores valores em 2015, evidenciando que, em período de estiagem, produziu-se uma mesma quantidade de biomassa que nos outros anos, porém com menor evapotranspiração, isso torna-a um potencial indicador de estresse hídrico e de seca. 


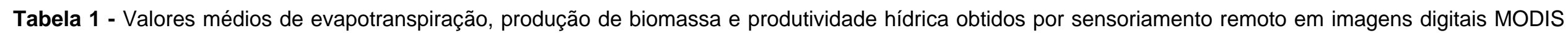
entre 2013 e 2015 em perímetro urbano (Urb), perímetro rural na macroregião de Ribeirão Preto (RurRP) e perímetro rural na macroregião do Alto Paranapanema (RurPA)

\begin{tabular}{|c|c|c|c|c|c|c|c|c|c|c|c|c|c|c|c|c|c|c|c|c|c|}
\hline \multirow[b]{3}{*}{$\begin{array}{c}\text { Dia } \\
\text { Juliano }\end{array}$} & \multicolumn{9}{|c|}{ Evapotranspiração (mm dia $\left.{ }^{-1}\right)$} & \multicolumn{6}{|c|}{ Biomassa $\left(\mathrm{kg} \mathrm{ha}^{-1} \mathrm{dia}^{-1}\right)$} & \multicolumn{6}{|c|}{ Produtividade Hídrica $\left(\mathrm{kg} \mathrm{m}^{-3}\right)$} \\
\hline & \multirow[b]{2}{*}{ Urb } & \multirow{2}{*}{$\begin{array}{c}2013 \\
\text { RurR } \\
\text { P }\end{array}$} & \multirow{2}{*}{ RurPA } & \multicolumn{3}{|c|}{2014} & \multicolumn{3}{|c|}{2015} & \multicolumn{2}{|c|}{2013} & \multicolumn{2}{|c|}{2014} & \multicolumn{2}{|c|}{2015} & \multicolumn{2}{|c|}{2013} & \multicolumn{2}{|c|}{2014} & \multicolumn{2}{|c|}{2015} \\
\hline & & & & Urb & $\begin{array}{c}\text { RurR } \\
\mathbf{P}\end{array}$ & $\begin{array}{c}\text { RurP } \\
\text { A }\end{array}$ & Urb & $\underset{P}{\text { RurR }}$ & $\begin{array}{c}\text { RurP } \\
\text { A }\end{array}$ & RurRP & RurPA & RurRP & RurPA & RurRP & RurPA & $\begin{array}{c}\text { RurR } \\
\text { P }\end{array}$ & RurPA & $\begin{array}{c}\text { RurR } \\
\text { P }\end{array}$ & $\begin{array}{c}\text { RurP } \\
\text { A }\end{array}$ & $\begin{array}{c}\text { RurR } \\
\mathbf{P}\end{array}$ & $\begin{array}{c}\text { RurP } \\
\text { A }\end{array}$ \\
\hline 1 & 1.13 & 3.51 & 4.14 & 1.06 & 3.29 & 3.57 & 0.49 & 1.51 & 1.64 & 84.83 & 106.04 & 78.22 & 83.32 & 37.77 & 37.77 & 3.06 & 3.83 & 3.25 & 3.94 & 3.64 & 4.45 \\
\hline 17 & 1.06 & 3.30 & 3.89 & 1.13 & 3.51 & 3.82 & 1.01 & 3.14 & 3.40 & 78.14 & 97.67 & 86.20 & 91.83 & 76.31 & 76.31 & 3.16 & 3.94 & 3.38 & 4.10 & 3.51 & 4.30 \\
\hline 33 & 0.95 & 2.94 & 3.47 & 0.92 & 2.87 & 3.12 & 1.02 & 3.18 & 3.45 & 72.32 & 90.40 & 69.68 & 74.23 & 77.61 & 77.61 & 3.28 & 4.09 & 3.30 & 4.01 & 3.51 & 4.30 \\
\hline 49 & 1.04 & 3.25 & 3.83 & 0.94 & 2.91 & 3.16 & 1.04 & 3.25 & 3.53 & 82.72 & 103.40 & 72.02 & 76.71 & 84.46 & 84.46 & 3.44 & 4.30 & 3.37 & 4.09 & 3.77 & 4.62 \\
\hline 65 & 0.82 & 2.56 & 3.02 & 0.88 & 2.74 & 2.98 & 0.71 & 2.21 & 2.40 & 64.23 & 80.28 & 71.76 & 76.44 & 53.05 & 53.05 & 3.34 & 4.17 & 3.59 & 4.36 & 3.17 & 3.88 \\
\hline 81 & 0.89 & 2.77 & 3.27 & 0.92 & 2.86 & 3.10 & 0.96 & 2.98 & 3.24 & 72.34 & 90.42 & 75.03 & 79.92 & 80.08 & 80.08 & 3.44 & 4.30 & 3.61 & 4.38 & 3.82 & 4.68 \\
\hline 97 & 1.06 & 3.30 & 3.89 & 0.75 & 2.34 & 2.54 & 0.95 & 2.95 & 3.21 & 84.30 & 105.37 & 64.11 & 68.29 & 80.08 & 80.08 & 3.42 & 4.28 & 3.77 & 4.58 & 3.92 & 4.80 \\
\hline 113 & 1.00 & 3.11 & 3.66 & 1.08 & 3.37 & 3.66 & 0.90 & 2.81 & 3.06 & 79.87 & 99.83 & 83.38 & 88.82 & 74.02 & 74.02 & 3.43 & 4.29 & 3.37 & 4.09 & 3.79 & 4.64 \\
\hline 129 & 0.76 & 2.35 & 2.77 & 0.57 & 1.76 & 1.92 & 0.82 & 2.55 & 2.77 & 60.90 & 76.13 & 60.90 & 64.88 & 65.26 & 65.26 & 3.44 & 4.30 & 3.48 & 4.23 & 3.65 & 4.46 \\
\hline 145 & 0.78 & 2.44 & 2.87 & 0.84 & 2.62 & 2.84 & 0.73 & 2.27 & 2.46 & 60.56 & 75.70 & 63.02 & 67.13 & 58.25 & 58.25 & 3.29 & 4.12 & 3.22 & 3.91 & 3.65 & 4.46 \\
\hline 161 & 0.77 & 2.39 & 2.82 & 0.71 & 2.21 & 2.40 & 0.78 & 2.42 & 2.63 & 69.77 & 87.22 & 55.38 & 59.00 & 62.84 & 62.84 & 3.43 & 4.29 & 3.33 & 4.05 & 3.69 & 4.52 \\
\hline 177 & 0.74 & 2.31 & 2.72 & 0.62 & 1.93 & 2.10 & 0.73 & 2.27 & 2.46 & 59.33 & 74.16 & 45.07 & 48.01 & 51.87 & 51.87 & 3.40 & 4.26 & 3.03 & 3.68 & 3.19 & 3.90 \\
\hline 193 & 0.86 & 2.67 & 3.15 & 0.82 & 2.56 & 2.78 & 0.73 & 2.27 & 2.46 & 58.96 & 73.70 & 49.14 & 52.34 & 54.68 & 54.68 & 2.88 & 3.60 & 2.55 & 3.10 & 3.35 & 4.10 \\
\hline 209 & 1.13 & 3.50 & 4.13 & 0.72 & 2.24 & 2.43 & 0.87 & 2.70 & 2.93 & 76.71 & 95.89 & 46.90 & 49.96 & 61.33 & 61.33 & 2.83 & 3.54 & 2.67 & 3.24 & 3.15 & 3.85 \\
\hline 225 & 0.90 & 2.80 & 3.30 & 0.61 & 1.90 & 2.07 & 0.80 & 2.49 & 2.71 & 54.65 & 68.31 & 34.47 & 36.72 & 51.09 & 51.09 & 2.40 & 3.00 & 2.26 & 2.75 & 2.77 & 3.40 \\
\hline 241 & 0.80 & 2.48 & 2.92 & 0.61 & 1.89 & 2.05 & 0.62 & 1.92 & 2.08 & 47.70 & 59.63 & 35.02 & 37.30 & 37.16 & 37.16 & 2.33 & 2.91 & 2.30 & 2.79 & 2.57 & 3.14 \\
\hline 257 & 0.67 & 2.09 & 2.46 & 0.43 & 1.34 & 1.46 & 0.43 & 1.33 & 1.44 & 39.43 & 49.29 & 23.29 & 24.80 & 26.84 & 26.84 & 2.24 & 2.81 & 2.13 & 2.59 & 2.80 & 3.43 \\
\hline 273 & 0.86 & 2.69 & 3.17 & 0.66 & 2.06 & 2.23 & 0.34 & 1.05 & 1.14 & 50.01 & 62.51 & 37.37 & 39.81 & 21.17 & 21.17 & 2.25 & 2.82 & 2.27 & 2.76 & 2.83 & 3.47 \\
\hline 289 & 1.00 & 3.12 & 3.68 & 0.57 & 1.78 & 1.93 & 0.80 & 2.49 & 2.71 & 64.89 & 81.11 & 32.54 & 34.66 & 53.50 & 53.50 & 2.59 & 3.23 & 2.24 & 2.72 & 2.96 & 3.62 \\
\hline 305 & 0.96 & 2.98 & 3.51 & 0.83 & 2.59 & 2.81 & 0.74 & 2.31 & 2.51 & 61.20 & 76.50 & 50.81 & 54.13 & 50.91 & 50.91 & 2.59 & 3.23 & 2.52 & 3.06 & 3.08 & 3.77 \\
\hline 321 & 0.88 & 2.74 & 3.23 & 0.84 & 2.60 & 2.83 & 0.90 & 2.80 & 3.04 & 58.09 & 72.61 & 51.69 & 55.07 & 62.82 & 62.82 & 2.71 & 3.39 & 2.58 & 3.13 & 3.16 & 3.86 \\
\hline 337 & 1.04 & 3.25 & 3.83 & 0.90 & 2.79 & 3.02 & 0.94 & 2.93 & 3.18 & 74.35 & 92.94 & 61.13 & 65.12 & 69.53 & 69.53 & 2.94 & 3.68 & 2.92 & 3.55 & 3.38 & 4.14 \\
\hline 353 & 0.82 & 2.55 & 3.00 & 1.04 & 3.23 & 3.51 & 0.86 & 2.67 & 2.90 & 56.58 & 70.73 & 74.92 & 79.80 & 66.13 & 66.13 & 2.86 & 3.58 & 3.12 & 3.80 & 3.52 & 4.31 \\
\hline Média & 0.91 & 2.83 & 3.34 & 0.80 & 2.50 & 2.71 & 0.79 & 2.46 & 2.67 & 65.73 & 82.17 & 57.48 & 61.23 & 58.99 & 58.99 & 2.99 & 3.74 & 2.97 & 3.60 & 3.34 & 4.09 \\
\hline Máximo & 1.13 & 3.51 & 4.14 & 1.13 & 3.51 & 3.82 & 1.04 & 3.25 & 3.53 & 84.83 & 106.04 & 86.20 & 91.83 & 84.46 & 84.46 & 3.44 & 4.30 & 3.77 & 4.58 & 3.92 & 4.80 \\
\hline Mínimo & 0.67 & 2.09 & 2.46 & 0.43 & 1.34 & 1.46 & 0.34 & 1.05 & 1.14 & 39.43 & 49.29 & 23.29 & 24.80 & 21.17 & 21.17 & 2.24 & 2.81 & 2.13 & 2.59 & 2.57 & 3.14 \\
\hline Amplitude & 0.46 & 1.43 & 1.68 & 0.70 & 2.17 & 2.36 & 0.71 & 2.20 & 2.39 & 45.40 & 56.75 & 62.92 & 67.02 & 63.29 & 63.29 & 1.20 & 1.50 & 1.64 & 1.99 & 1.35 & 1.66 \\
\hline
\end{tabular}


Figura 3 - Comportamento temporal das médias da área de estudo da evapotranspiração (a), produção primária de biomassa (b) e produtividade hídrica (c) entre 2013 e 2015

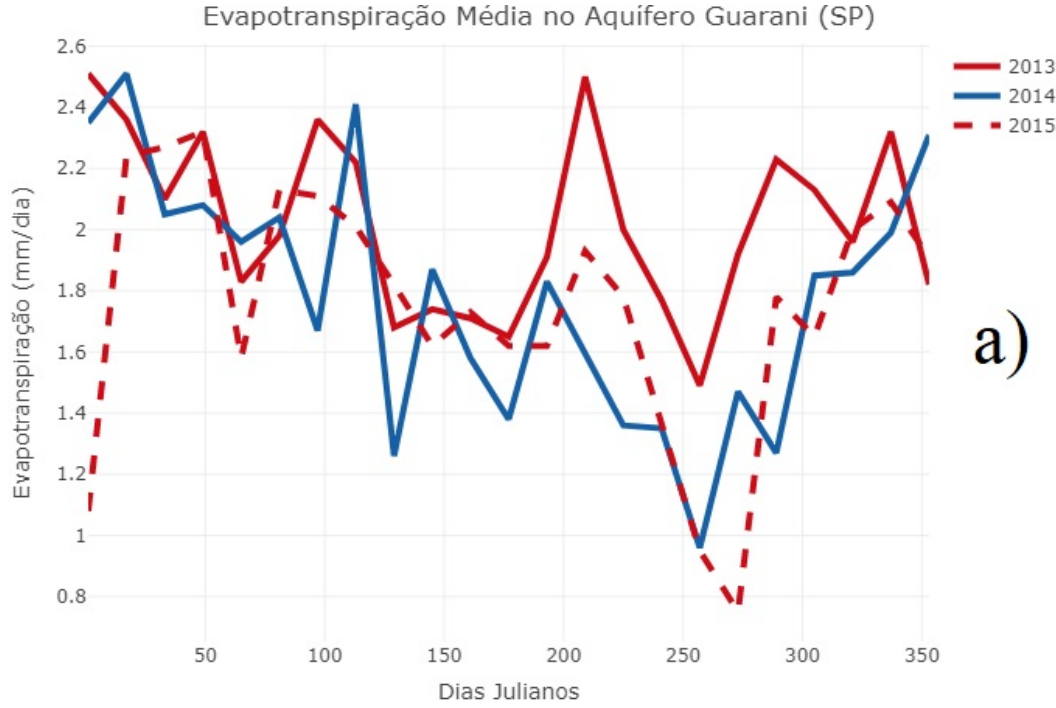

Produção Primária Média no Aquifero Guarani (SP)

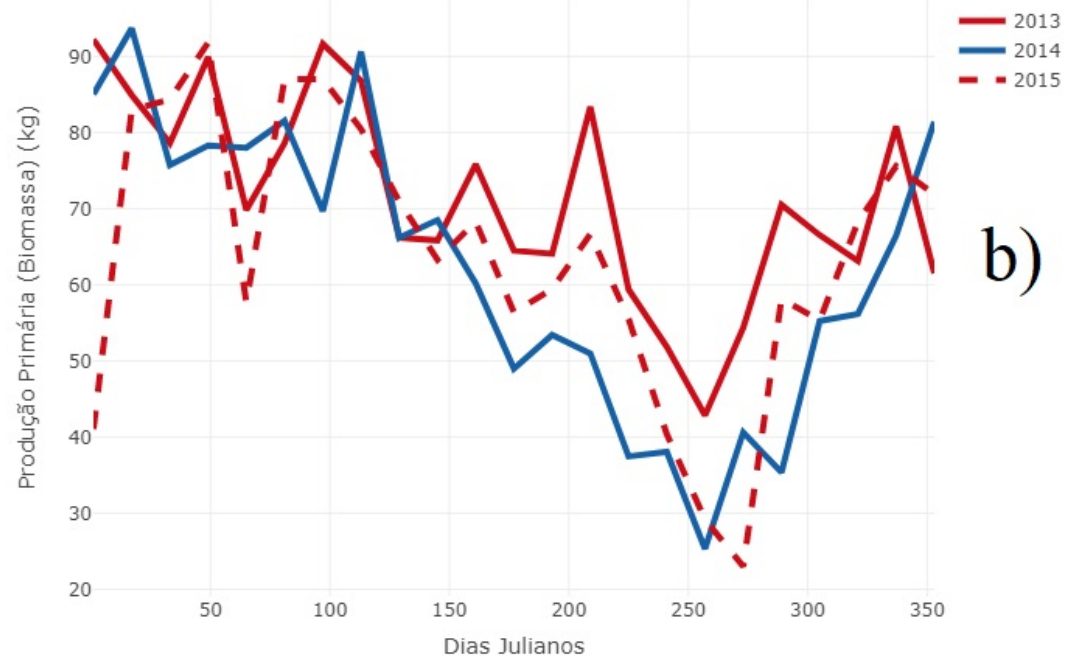

Produtividade Hidrica Média no Aquifero Guarani (SP)

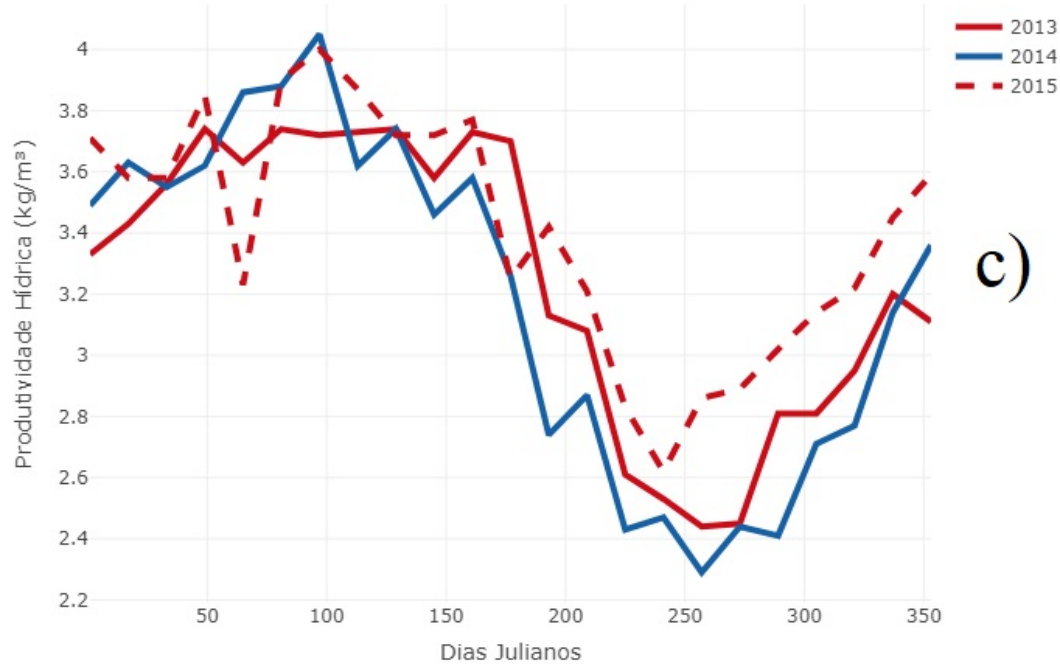


Figura 4 - Distribuição espacial da evapotranspiração, produção primária de biomassa e produtividade hídrica no dia juliano 273 de 2013, 2014 e 2015

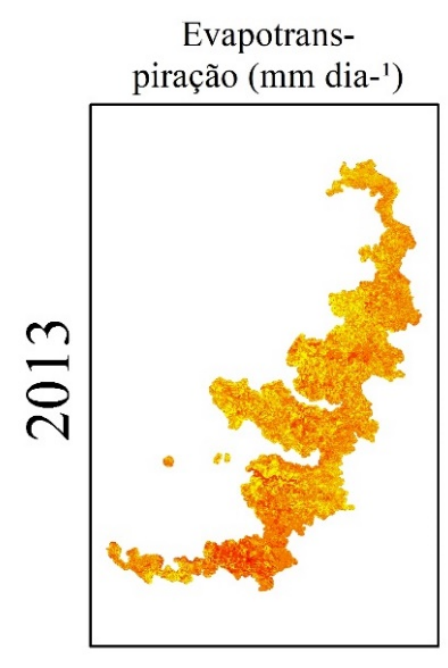

Produção de Biomassa ( $\left.\mathrm{kg} \mathrm{ha}^{-1} \mathrm{dia}^{-1}\right)$

Produtividade

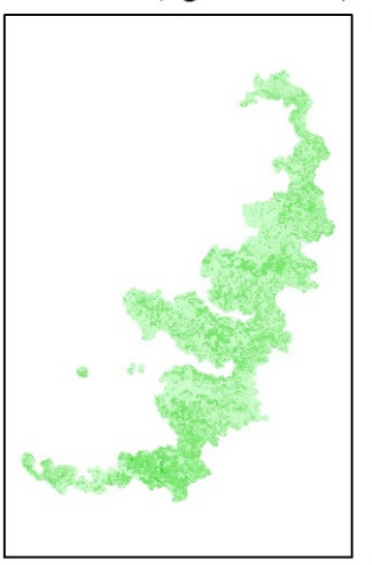
Hídrica $\left(\mathrm{kg} \mathrm{m}^{-3}\right)$
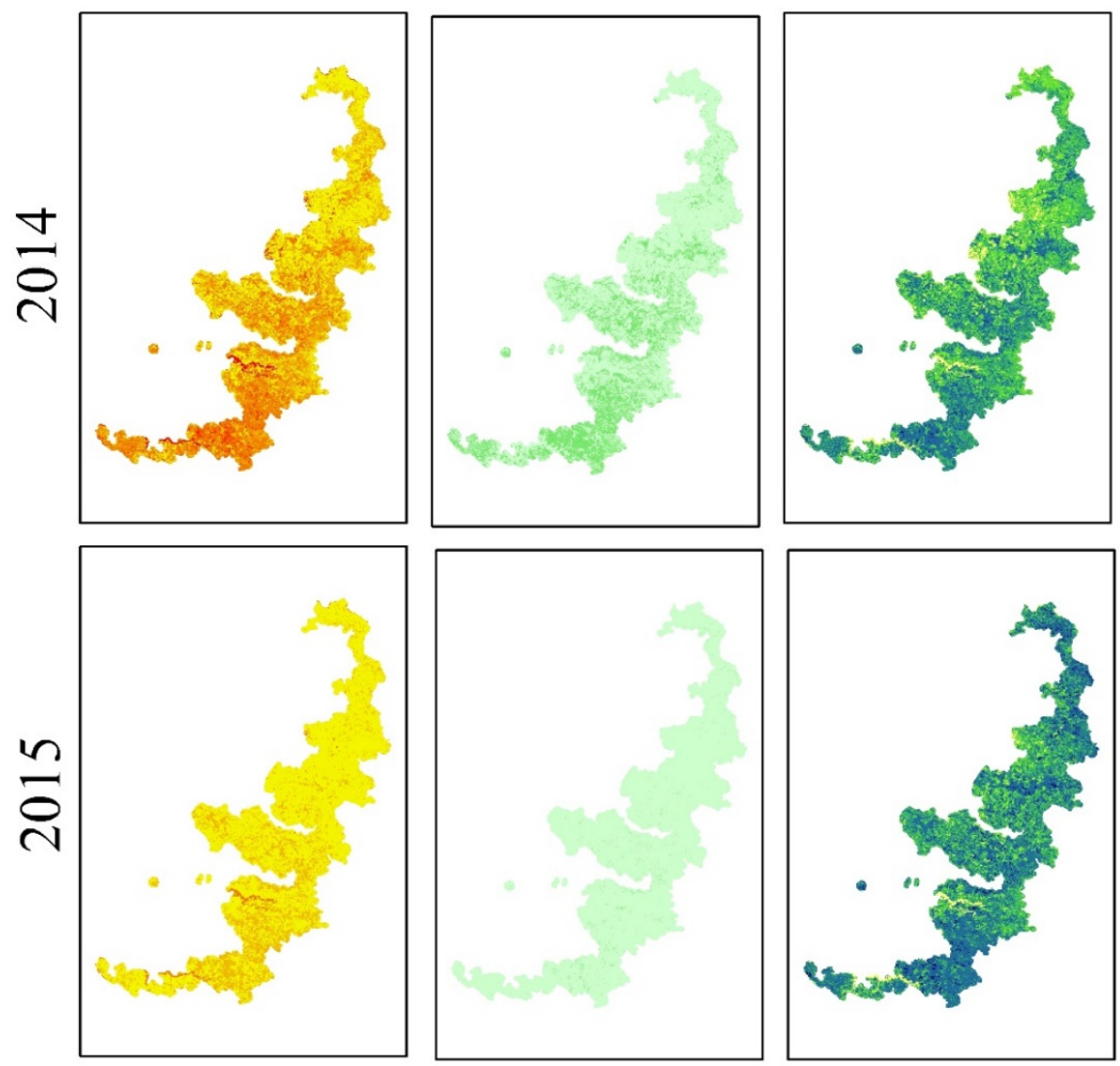

\section{Evapotranspiração}

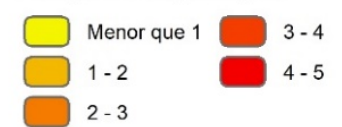

\section{Produção de Biomassa}

\section{Produtividade Hídrica}

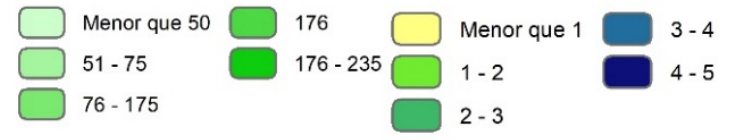<smiles>NC1CC2CC1CN2</smiles>

0 125 250 $500 \mathrm{~km}$ 


\section{CONCLUSÕES}

O monitoramento espacial de indicadores agrometereológicos, como a evapotranspiração real e a produção primária de biomassa, obtidas através dos modelos SAFER e Monteith, respectivamente, mostraram-se eficazes no diagnóstico de tendências sazonais em uma área com resolução espacial mínima de 250 metros. Esses parâmetros são úteis para realizar inferências sobre o comportamento da recarga de aquífero, levandose em conta a relação da recarga com o balanço hídrico (EILERS, 2004). Cabe ressaltar que esses resultados também são úteis à gestão ambiental em nível regional, já que a metodologia apresentada é aplicável à escala de propriedade rural e de distritos municipais (TEIXEIRA et al, 2016, TEIXEIRA et al, 2017; COAGUILA et al, 2017).

Foram encontradas evidências que a estiagem de 2014 teve maior impacto na região rural ao norte do afloramento paulista do Aquífero Guarani (região de Ribeirão Preto) em comparação à região do Paranapanema. Esse fato deve-se ao predomínio de plantio de cana-de-açúcar no norte do estado, enquanto no sul tem-se o predomínio de Eucaliptos. Em valores médios para toda área paulista do afloramento do Aquífero Guarani ocorreu diminuição da evapotranspiração e produção de biomassa nos anos de estiagem (20142015).

A produtividade hídrica, quando tomada em referência uma série histórica que permita a análise de tendência, é um potencial indicador de estresse hídrico de vegetação e culturas.

Essas informações geram subsídio para inferir sobre o comportamento da recarga de um sistema aquífero, necessário para a tomada de medidas visando a utilização sustentável das águas subterrâneas, evitando impactos ambientais negativos provenientes da superexplotação dos aquíferos. A utilização de ferramentas de sensoriamento remoto permite a análise espacializada do aquífero.

\section{REFERÊNCIAS}

ALBUQUERQUE FILHO, J. L.; CARVALHO, A. M.; BARBOSA, M. C.; IKEMATSU, P.; FREITAS, C. G. L.; MONTEIRO, A. C. M. C.; IRITANI, M.; PRESSINOTTI, M. M. N.; ROCHA, G., TAKASHI; SILVA, M. P. M.; KOVACS, A.; CAMARGO, G. G. Subsídios ao plano de desenvolvimento e proteção ambiental da área de afloramento do Sistema Aquífero Guarani no Estado de São Paulo. São Paulo: Instituto de Pesquisas Tecnológicas, 2011. 
Agência Nacional de Águas - ANA. Levantamento da agricultura irrigada por pivôs centrais no Brasil - 2014. Relatório Síntese. Brasília: ANA, 2016.

BASTIAANSSEN, W. G. M.; ALI, S. A new crop yield forecasting model based on satellite measurements applied across the Indus basin, Pakistan. Agriculture, Ecosystems \& Environment, v.94, p. 321-340, 2003.

BRUIN, H. A. R.; STRICKER, J. N. M. Evaporation of grass under non-restricted soil misture conditions. Hydrological Sciences Journal, v. 45, p.391-406, 2000.

COAGUILA, DANIEL N. ; HERNANDEZ, FERNANDO B. T. ; TEIXEIRA, A. H. DE C. ; FRANCO, RENATO A. M. ; LEIVAS, JANICE F. Water productivity using SAFER: simple algorithm for evapotranspiration retrieving in watershed. Revista Brasileira de Engenharia Agrícola e Ambiental, v. 21, p. 524-529, 2017.

DOBROVOLSKI, R.; RATTIS, L. Water Collapse in Brazil: the danger of relying on what you neglect, Natureza e Conservação, v. 3, ed. 1, jan./jun. 2015, p. 80-3.

EILERS, V. H. M. Estimativa de Recarga de Águas Subterrâneas utilizando o Método do Balanço Hídrico. In: CONGRESSO BRASILEIRO DE ÁGUAS SUBTERRÂNEAS, 13., 2004. Anais... São Paulo, 2004. v. 01. p. 1-20.

ESPINOZA, J. C. et al. The Extreme 2014 Flood in South-Western Amazon Basin: The Role of Tropical-Subtropical South Atlantic SST Gradient, Environmental Research Letters, n.9, 2014.

IBGE. Instituto Brasileiro de Geografia e Estatística. Disponível em <https://www.ibge. gov.br/>. Acesso 13 jan. 2018.

INSTITUTO NACIONAL DE PESQUISAS ESPACIAIS (INPE). Divisão de Sensoriamento Remoto (DSR). Canasat - Mapeamento da cana via imagens de satélite de observação da terra. 2013. Disponível em <http://www.dsr.inpe.br/laf/canasat/index.html>. Acesso 3 mar. de 2018.

MANZIONE, R. L.; SOLDERA, B. C.; PAES, C. O.; WENDLAND, E. C. Analyses of water table depths variations in an outcrop area of the guarani aquifer system in Brotas/SP-Brazil. Irriga, v. 1, p. 186-197, 2015.

MANZIONE, R. L.; SOLDERA, B. C.; WENDLAND, E. C. Groundwater system response at sites with different agricultural land uses: case of the Guarani Aquifer outcrop area, Brotas/SP-Brazil. Hydrological Sciences Journal, v. 62, p. 28-35, 2016.

MAPBIOMAS. Coleções MapBiomas. Disponível em <http://mapbiomas.org/pages/database/ mapbiomas_collection>. Acesso 3 mar. 2018.

NAVA, A.; MANZIONE, R. L. Resposta de niveis freáticos do sistema Aquifero Bauru (Formação Adamantina) em função da precipitação e evapotranspiração sob diferentes usos da terra. Águas Subterrâneas, v. 29, p. 191, 2015.

NOBRE, C. et al. The Record Drought and Water Crises of Summer 2014 in Southeastern Brazil, Bulletin of the American Meteorogical Society, 2015

RAUPACH, M. R. Combination theory and equilibrium evaporation. Quarterly Journal of the Royal Meteorological Society, v.127, p.1149-1181, 2001. 
REISSLER, J. ; MANZIONE, R. L. Classificação do uso da terra em área de afloramento do Sistema Aquífero Guarani entre 2002 e 2011: o caso da bacia do Ribeirão do Jacú, Tejupá/SP. Águas Subterrâneas, v. 30, p. 172-189, 2016.

RODRIGUES, S. B. S. ; MANTOVANI, E. C.; OlIVEIRA, R. A.; PAIVA, H. N.; ALVES, M. E. B. M. E. B. Necessidades Hídricas de Mudas de Eucalipto na Região Centro-Oeste de Minas Gerais. Irriga, v. 16, n. 2, p. 212-223, 2011.

ROMAGNOLI, I. ; MANZIONE, R. L. . Expansão do Agrohidronegócio no Pontal do Paranapanema (UGRHI-22): Ocupação da Terra pela Cultura da Cana-De-Açúcar entre os anosde 2002 e 2013 x Vulnerabilidade de Aquíferos. In: CONGRESSO BRASILEIRO DE ÁGUAS SUBTERRÂNEAS, 19., 2016. Anais... Campinas, 2016.

SANTAROSA, L. V.; MANZIONE, R. L . Modelo Hidrogeológico Conceitual da Estação Ecológica de Santa Bárbara (EEcSB) em área do Sistema Aquífero Bauru (SAB). Águas Subterrâneas, v. 31, p. 404-412, 2017.

SILVA, R. C. F.; MANZIONE, R. L. Aplicação do modelo Hartt no estudo das oscilações dos níveis freáticos do Sistema Aquífero Bauru (SAB) sob vegetação de cerrado. Águas Subterrâneas, v. 30, p. 362-374, 2016.

TEIXEIRA, A. H. C., SCHERER-WARREN, M., HERNANDEZ, F. B. T., ANDRADE, R.G., LEIVAS, J.F. Large-Scale Water Productivity Assessments with MODIS Images in a Changing Semi-Arid Environment: A Brazilian Case Study. Remote Sensing, v. 5, p. 5783-5804, 2013.

TEIXEIRA, A. H. C. Determining Regional Actual Evapotranspiration of Irrigated Crops and Natural Vegetation in the Sâo Francisco River Basin (Brazil) Using Remote Sensing and Penman-Monteith Equation. Remote Sensing, n. 2, v. 4, p. 1287-1319, 2010.

TEIXEIRA, A. H. C.; LEIVAS, J. F.; RONQUIM, C. C.; VICTORIA, D. C. Sugarcane Water Productivity Assessments in the São Paulo state, Brazil. International Journal of Remote Sensing Applications, v. 6, p. 84-95, 2016.

TEIXEIRA, A. H. C.; BASTIAANSSEN, W. G. M.; AHMAD, M.; BOS, M. G. Reviewing SEBAL input parameters for assessing evapotranspiration and water productivity for the Low-Middle São Francisco River basin, Brazil Part A: Calibration and validation. Agricultural and Forest Meteorology, v. 149, p. 462-476, 2009.

TEIXEIRA, A. H. C.; LEIVAS, J. F.; SILVA, G. B.. Options for using Landsat and RapidEye satellite images aiming the water productivity assessments in mixed agro-ecosystems. Proceedings of SPIE, v. 9998, p. 99980A-1-99980A-11, 2016.

TEIXEIRA, A. H. C. ; LEIVAS, J. F. ; HERNANDEZ, F. B. T. ; FRANCO, R. A. M. . Large-scale radiation and energy balances with Landsat 8 images and agrometeorological data in the Brazilian semiarid region. Journal of Applied Remote Sensing, v. 11, p. 016030-1-016030-15, 2017. 